\title{
Initial Code Acquisition in the Cooperative Non-coherent MIMO DS-CDMA Downlink
}

\author{
SeungHwan Won, Student Member, IEEE, Kyungchun Lee and Lajos Hanzo, Fellow, IEEE
}

\begin{abstract}
In this paper we investigate a realistic code acquisition assisted cooperative Non-Coherent (NC) Multiple-Input Multiple-Output (MIMO) DS-CDMA downlink scenario, when communicating over uncorrelated single-path and multi-path Rayleigh channels. The probabilities of correct detection and false alarm have been derived analytically. Furthermore, a Mean Acquisition Time (MAT) formula is provided for the cooperative transmission scenario considered. The associated MAT performance trends are characterised as a function of both the number of Relay Stations (RSs), as well as that of the receive antennas and link imbalance. As opposed to the classic scenario of having co-located MIMO elements, our findings suggest that employing distributed MIMO elements acting as RSs combined with multiple receive antennas leads to an improved MAT performance.
\end{abstract}

Index Terms-DS-CDMA, code acquisition, relay station, cooperative, non-coherent MIMO system.

\section{INTRODUCTION}

In wireless mobile networks, fading constitutes one of the main sources of channel-induced impairment. A powerful technique of overcoming the fading imposed by multi-path propagation is constituted by spatial diversity invoking multiple transmit/multiple receive antennas, which has attracted considerable research interests [1], [2]. Furthermore, the substantial appeal of MIMOs is that their capacity increases linearly with the Signal-to-Interference plus Noise Ratio (SINR), as opposed to the more modest logarithmic increment of the classic Shannon-Hartley law, which may be readily elucidated by assigning the increased transmit power to an additional antenna and therefore linearly increasing the throughput [3]. However, in realistic propagation environments, the multiple antenna's signals typically become correlated owing to the size-limitation of the Mobile Station (MS) and Base Station (BS). Hence the spatial diversity gain of independently faded signals is often eroded. This phenomenon is typically imposed by shadow fading. In order to cope with this problem, various cooperative and RS-aided transmission schemes have been proposed [4] - [6]. In low-complexity cooperative systems a MS receives the two-hop DownLink (DL) signal both via the RS as well as the directly detected DL signal of the BS. Since these two signals generally arrive through completely different - rather than correlated - propagation paths, cooperative transmission becomes capable of mitigating the above-mentioned correlated shadow fading effects. Furthermore, exploiting the intermediate RS, cooperative transmission has the potential of extending the cell area and/or of improving the quality of cell-edge coverage, which results in requiring a reduced number of BSs. The RS in cooperative systems filters the signal received from the BS and retransmits it to the MS. The relaying schemes are commonly classified into two types: Amplify-and-Forward (AF) as well as Decode-and-Forward (DF) regimes [5], [6]. In the AF scheme, the RS simply retransmits the scaled version of the encountered signal and hence the noise component may also be amplified. By contrast, the DF aided RS fully decodes the received signal and forwards the re-encoded version in order to avoid the noise amplification. However, the DF strategy undoubtedly increases the complexity of RSs. In cooperative or distributed MIMO scenarios, the RS may be constituted by an intermediate MS that is currently not engaged in active communication or by a fixed RS that is installed at a specific position in a cell. However, for the sake of creating high-reliability RSs, in this treatise we consider fixed RSs [6]. It is also assumed that there is a Line-Of-Sight (LOS) path between the RS as well as the BS [6] and that the required initial procedures have been completed, before commencing the stage of RS-aided code acquisition.

In inter-cell synchronous CDMA systems the MS's receiver must be capable of synchronously aligning a locally generated PseudoNoise (PN) code with the received composite multiuser signal containing the desired PN sequence within an allowable timing error. Our analysis addresses the initial acquisition stage of CDMA DL systems, aiming for the acquisition of the coarse timing of the signals received. During the initial acquisition stage, the MS is unable to employ any channelrelated information. Therefore, the received signals can only be non-coherently combined by employing equal gain combining, rather than using selection combining, as suggested in [9]. Hence our aim is to minimise the MAT, which is directly proportional to both the correct detection probability $\left(P_{D}\right)$ as well as to the time required by the acquisition scheme to notice after the elapse of the code phase verification period that a false locking event occurred and then to return to the search mode. The uncertainty region (or search window width) in the DL corresponds to the entire period of the PN sequence, which tends to be quite wide, namely on the order of the code length, such as $\left(2^{15}-1\right)$ chip intervals in the inter-cell synchronous CDMA DL [10], [11]. Accordingly, the MAT is minimised in terms of serial search techniques by achieving the best possible $P_{D}$, whereas maintaining as low a value of the false alarm probability $\left(P_{F}\right)$ and false locking penalty as possible. Substantial research efforts have been devoted to the design of code acquisition techniques optimised for SingleInput Single-Output (SISO) scenarios [12], [13]. These efforts have also been extended to code acquisition schemes designed for MIMO systems having co-located antenna elements [14] 
- [16]. These results suggest that increasing the number of transmit antennas in a NC MIMO-aided CDMA system having co-located antenna elements typically results in a MAT performance degradation, due to sharing the total transmit power across several MIMO elements, regardless whether single-path or multi-path scenarios are considered [14] - [16], even if the associated degradation is partially compensated by increasing the number of receiver elements. Since there are no in-depth studies representing the fundamental characteristics of code acquisition schemes in RS-aided scenarios in the context of multi-path propagation scenarios, solving this open problem is the objective of the present contribution. Against this background, in this treatise we investigate serial search based code acquisition schemes designed for fixed RS-aided scenarios. More explicitly, we quantify both the attainable $P_{D}$ as well as the $P_{F}$ performance as a function of both the SINR per chip $\left(E_{c} / I_{0}\right)$ and that of the number of receive antennas $R$. Furthermore, we characterise the attainable MAT versus $E_{c} / I_{0}$ performance parameterised by the grade of link imbalance as well as by the number of receive antennas and RSs in both single- and multi-path scenarios.

This paper is organised as follows. Section II describes the system investigated, followed by the correct detection and false alarm probability analysis of code acquisition schemes in the context of uncorrelated Rayleigh channels, whereas the MAT analysis of fixed RS-aided scenarios is illustrated in Section III. In Section IV, our numerical MAT results are discussed, whilst our conclusions are offered in Section V.

\section{CORRECT DETECTION AND FALSE ALARM PROBABILITY}

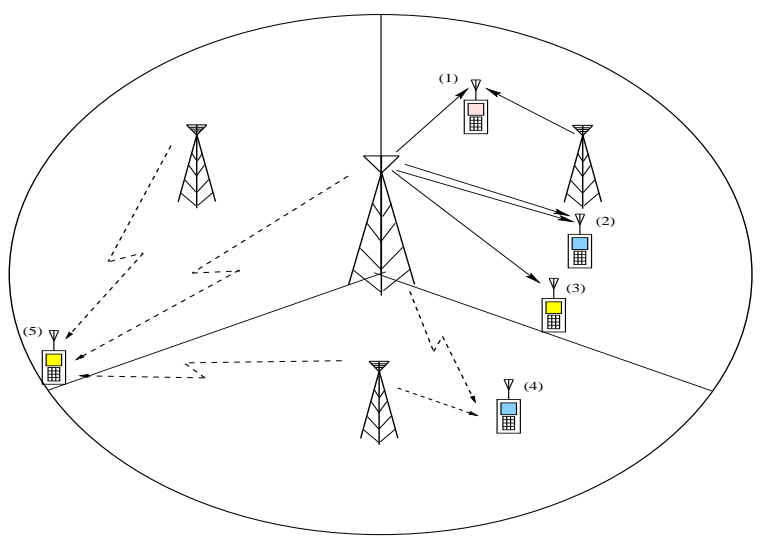

Fig. 1. Code acquisition scenarios in cooperative MIMO environments, which encompasses three normalised-power scenarios (denoted as (1), (2) and (3)) and two increased-power ones (denoted as (4) and (5)) when considering one or two RSs.

First of all, our three-stage code acquisition scheme employed in RS-aided inter-cell synchronous DS-CDMA DL systems is summarised as follows:

- First stage: Timing acquisition between the BS and master RSs.

$\Rightarrow$ Both are capable of extracting timing information from the received signal because they are fixed and it is already known which one was assigned a specific WalshHadamard code for its future communications.

- Second stage: Timing acquisition between the BS and master RSs as well as MSs.

$\Rightarrow$ In this scenario, the MSs are capable of benefitting from cooperative diversity. This scenario will be investigated in our analysis.

- Third stage: Timing acquisition among the RSs (master and slave) and MSs.

$\Rightarrow$ By employing a signalling message termed as $M_{1}$ in the uplink, the slave RSs and the MSs are registered with a specific master RS. When using another signalling message termed as $M_{2}$ among the slave RSs and the MSs, timing acquisition is accomplished within a previously established cluster. A new broadcast downlink channel is used for distributing the cluster assignment or grouping information. More specifically, the grouping information includes assignment of slave RSs and unique identification information for each element of the cluster. Based on the information provided by the broadcast channel, the $M_{2}$ messages are exchanged in order to establish timing acquisition among the constituents of a specific cluster.

Our analysis in this treatise is essentially confined to the second stage designed for employment in an RS-aided code acquisition scheme. Fig.1 illustrates a number of code acquisition scenarios encountered in fixed RS-aided environments, when considering one or two RSs. The traffic cell of Fig.1 is divided into three sectors and a fixed RS is employed in each sector. We assume that both the BS and the RS of Fig.1 have a single transmit antenna, except for the co-located MIMO-element scenario having two transmit antennas. It is also assumed that both the BS and the master RSs are capable of supporting handovers and that the master RSs are capable of supporting both AF, DF as well as pilot transmission. Furthermore, we also assume that the slave RSs are capable of supporting both AF, DF as well as signalling message transmissions for the RS-aided cluster, while other slave RSs may only support AF and signalling message transmission and that only low-speed MSs can be included in a cluster aided by slave RSs. The top right sector of Fig. 1 depicts three scenarios, where the total allocated power is equally shared by the transmit antennas in both the co-located and cooperative transmission scenarios (termed as 'normalised-power scenario' ${ }^{1}$ ) as follows:

- Scenario (1) of Fig.1 : The MS receives the DL signals from both the RS located at the same sector's centre as well as from the BS and processes them in order to attain reliable code acquisition.

- Scenario (2) of Fig.1 : A co-located scenario having two transmit antennas is considered.

- Scenario (3) of Fig.1 : A SISO scenario is used as a benchmarker.

By contrast, in both the fourth and fifth scenarios it is assumed that the total allocated power is also proportionately increased according to the number of RSs (referred to as 'increased-

\footnotetext{
${ }^{1}$ This means that a fraction of $1 / P$ of the total power is assigned to the BS and RSs, respectively. The total power allocated becomes unity.
} 
power scenario' ${ }^{2}$ ), as follows:

- Scenario (4) of Fig.1 : It is exactly the same as the first one, except for its different power allocation.

- Scenario (5) of Fig.1 : When the MS is located at the edge of the top left sector of Fig.1, the signal received from the RS at the adjacent sector's centre seen at the bottom of Fig.1 also arrives at the MS at a substantial signal strength.

Furthermore, in the fifth case, this particular MS has to combine the signals received from all the three transmitters for the sake of reliable code acquisition. The average strength of the RS' signal varies depending on the location of the MS. Explicitly, when the MS is located near the cell-edge, the signal received from the RS generally has a higher signal strength with respect to that of the BS, which results in the phenomenon often referred to as link imbalance between the signals received by the MS.

We assume that a finite-length tapped delay line channel model generates $L$ independently Rayleigh-faded multi-path signals, each arriving with a time delay $\tau_{l}$ having a tap spacing of one chip-duration [17], [18], where $l=L$ is the number of multi-path components encountered. It is also assumed that the Rayleigh fading is sufficiently slow for the faded envelope to remain constant over the duration of $\tau_{D}=N \cdot T_{c}$, but sufficiently rapid so that the consecutive $\tau_{D}$-duration segments may be considered essentially independently faded, as in [10], [19], where $\tau_{D}$ indicates the integral dwell time, $N$ represents the number of chips accumulated over the duration of $\tau_{D}$ and $T_{c}$ is the chip duration. Furthermore, the Neyman-Pearson criterion [10], [20] is adopted, which leads to a constant falsealarm rate. This is because as a benefit of normalising the received signal by the background noise variance estimate, the fading channel's attenuation no longer affects the outcome of a test, regardless whether the desired signal is present or absent. The resultant scenario and the related test becomes reminiscent of an Additive White Gaussian Noise (AWGN) scenario. Consequently, in line with the findings of [10], [19], the mobile channel only affects the $P_{D}$ performance. In case of a co-located MIMO scenario the spacing of the multiple transmit elements at the $\mathrm{BS}$ is deemed to be $10 \lambda$ and that of the multiple receive elements at the MS is assumed to be $0.5 \lambda$ [21]. Accordingly, it is assumed that there is no spatial correlation among the DL signals emanating from the co-located $P$ transmit antennas. On the other hand, in case of a cooperative MIMO scenario the spacing of the multiple receive elements at the MS is also assumed to be $0.5 \lambda$ [21]. Finally, we note that the analysis of our code acquisition schemes is valid for both space-time coding [1] and for spatial division multiplexing [22] MIMOs.

The fixed RS-aided DS-CDMA DL signal received over the multi-path Rayleigh fading channel considered may be

\footnotetext{
${ }^{2}$ This implies that every diversity component generated in a RS-aided acquisition scenario, namely the BS and the RSs, has a unity power. Each component's power was the same as the total power of the normalisedpower scenario. Accordingly, when using $p^{\text {th }}$ order diversity, the total power allocated becomes $P$.
}

expressed as [14]

$$
\begin{aligned}
& r(t)=\sum_{p=1}^{P} \sum_{l=1}^{L} \sum_{r=1}^{R}\left[\alpha_{(p, l, r)} \sqrt{\frac{1}{P}} \sqrt{\frac{E_{c}}{T_{c}}} c\left(t+d T_{c}+\tau_{p}+\tau_{l}\right)\right. \\
& \left.\cdot w_{p}\left(t+d T_{c}+\tau_{p}+\tau_{l}\right) \cdot \exp \left(2 \pi f t+\phi_{(p, l, r)}\right)+I_{(p, l, r)}(t)\right],
\end{aligned}
$$

where $p=P$ is the number of $\mathrm{RSs}$ and $\mathrm{BS}, r=R$ is the number of receive antennas, and $\alpha_{(p, l, r)}$ represents the complex-valued envelope of the $(p, l, r)^{t h}$ signal path obeying the Rayleigh distribution. Furthermore, $E_{c}$ denotes the pilot signal energy per PN code chip, $c(t)$ represents a common PN sequence having a cell-specific code-phase offset, while $d$ is the code phase offset with respect to the phase of the local code. Still considering Eq. $1, w_{p}(t)$ identifies the specific Walsh code assigned to the $p^{t h} \mathrm{RS}, \tau_{p}$ indicates the relative time difference of the signal received from a RS with respect to the signal received from the BS, $f$ is the carrier frequency and finally, $\phi$ is the carrier phase of a specific user's modulator. Furthermore, $I_{(p, l, r)}(t)$ is the complex-valued AWGN having a double-sided power spectral density of $I_{0}$ at the $(p, l, r)^{t h}$ path. It is also noted that in the normalised-power scenario a factor denoted as $\sqrt{\frac{1}{P}}$ must be included in Eq. 1 due to equally sharing the total allocated power. On the other hand, this factor must be omitted in the increased-power scenario. For the sake of a fair comparison, it is assumed that in the absence of link imbalance, the total power received by the MS in RS-aided scenarios is the same as that of the corresponding multiple transmit antenna aided scenario for the normalisedpower scenario. In the increased-power scenario, it is assumed that in the absence of a link imbalance, the total power received by the MS in $\mathrm{RS}$-aided scenarios is the same as that of the corresponding multiple receive antenna assisted scenarios.

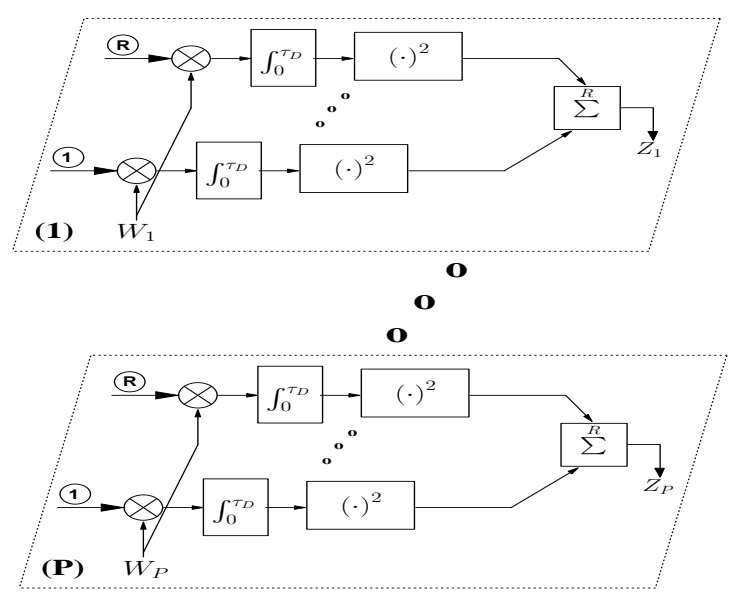

Fig. 2. Receiver structure of a NC code acquisition system employing $R$ receive antennas in $\mathrm{RS}$-aided scenarios.

Fig.2 depicts the block diagram of the NC DS-CDMA DL receiver designed for our code acquisition scheme using $R$ co-located receive antennas, where the first dotted box at the top of Fig.2 can be considered to be a single transmit and multiple receive antenna-aided receiver. Additionally, the related co-located multiple transmit, multiple receive antenna aided schematic is detailed in [14]. The receiver generates a decision variable by accumulating $R$ number of independently 
faded signals observed over a time interval for the sake of improving the $P_{D}$ in the mobile channel imposing both fading and poor SINR conditions. In order to simplify the receiver's structure, we omitted the front-end down converter, the chipmatched filter, as well as the sampler and descrambler of the PN code. Further details on the related schemes may be found in [23]. Let us now consider the effects of both the timing errors $\tau$ and those of the total frequency mismatch $\Delta f_{t}$ on the received signal. The timing errors are imposed by both the delay of the DL signal received from a mobile channel and by the sampling inaccuracy caused by having a finite search step size of $\Delta=T_{c} / 2$. The total frequency mismatch $\Delta f_{t}$ is the sum of the clock-drift-induced frequency mismatch $\Delta f_{m}$ between the BS transmitter and the MS's receiver, as well as of the effect of the Doppler shift, $\Delta f_{d}$. The term $\left(E_{c} / I_{0}\right)^{\prime}$ is defined as $\left(E_{c} / I_{0}\right) \cdot \operatorname{sinc}^{2}\left(\frac{\tau}{T_{c}}\right) \cdot \operatorname{sinc}^{2}\left(N \Delta f_{t} T_{c}\right)$ [16]. By employing the procedures proposed in [14], [23] in the context of the receiver structure of Fig.2, the final decision variable may be written as

$Z_{(k, p, l)}=\sum_{r=1}^{R}\left\|\frac{1}{\sqrt{2}} \cdot\left(\sqrt{\frac{4 E_{c}}{N I_{0}}} \cdot S_{(k, p, l)(r)}+I_{(k, p, l)(r)}\right)\right\|^{2}$

where $k$ denotes the $k^{t h}$ chip's sampling instant, $Z_{(k, p, l)}$ is a decision variable of the $(p, l)^{t h}$ path, which constitutes an element of the final decision variables, $Z_{1}, \ldots, Z_{P}$ in Fig.2, and $S_{(k, p, l)(r)}=\frac{1}{T_{c}} \int_{0}^{N T_{c}} c(t) c\left(t+d T_{c}+k T_{c}\right) \cdot \exp \left(j 2 \pi N \Delta f_{t}\right) d t$. If the PN codes have ideal AutoCorrelation Functions (ACFs), where the ACF has identical sidelobes to those of maximum length shift register sequences [10], $S_{(k, p, l)(r)}$ can be expressed as $N \cdot \exp \left(j 2 \pi N \Delta f_{t}\right)$ for the signal being present. On the other hand, in case of the signal being absent, it can be shown to be $-1 \cdot \exp \left(j 2 \pi N \Delta F_{t}\right)$. Therefore, $S_{(k, p, l)(r)}$ becomes deterministic [14], [23], while $I_{(k, p, l)(r)}$ is the complexvalued AWGN having zero means and variances of $\sigma^{2}=2$ for both their real and imaginary parts. Furthermore, $\|\cdot\|^{2}$ represents the Euclidean norm of the complex-valued argument and the factor of $1 / \sqrt{2}$ is employed to appropriately normalise the noise variance. Accordingly, the decision variable $Z_{(k, p, l)(r)}$ obeys a non-central chi-square Probability Density Function (PDF) with $2 R$ degrees of freedom, where the non-centrality parameter $\lambda_{x}$ is either $2 N\left(\frac{E_{c}}{I_{0}}\right)$ for the hypothesis of the desired signal being present $\left(H_{x}, x=1\right)$ or $\frac{2}{N}\left(\frac{E_{c}}{I_{0}}\right)$ for it being absent $\left(H_{x}, x=0\right)$ [14]. This PDF is given by [18]

$f_{Z_{(k, p, l)(r)}}\left(z \mid H_{x}\right)=\frac{1}{2} \cdot \exp \left[-\frac{\left(z+\lambda_{x}\right)}{2}\right] \cdot \mathcal{I}_{0}\left(\sqrt{z \cdot \lambda_{x}}\right)$,

where $z \geq 0, x=0$ or 1 and $\mathcal{I}_{0}(\cdot)$ is the zero ${ }^{t h}$-order modified Bessel function. Our aim is now that of expressing the PDF of a desired user's signal at the output of the acquisition scheme conditioned on the presence of the desired signal in $f_{Z_{(k, p, l)}}\left(z \mid H_{x}\right)$ derived for transmission over a spatially uncorrelated Rayleigh channel. In this scenario $E_{c}$ is multiplied by the square of the Rayleigh-distributed fading amplitude, $\beta$, which exhibits a chi-square distribution having two degrees of freedom and it is hence expressed as $f(\beta)=\frac{e^{-\beta / \sigma^{2}}}{\sigma^{2}}$, where $\sigma^{2}$ is the variance of the constituent Gaussian distribution.
Then the average pilot signal energy $\overline{E_{c}}$ per PN code chip can be expressed as $\overline{E_{c}}=\bar{\beta} E_{c}=\sigma^{2} E_{c}$ [10]. Therefore first the PDF $f_{Z_{(k, p, l)(r)}}\left(z \mid H_{x}, \beta\right)$ corresponding to $\beta$ conditioned on the hypothesis of the desired signal being transmitted over an AWGN channel having this specific SINR is weighted by the probability of occurrence $f(\beta)$ of encountering $\beta$, as quantified by the PDF. The resultant product is then averaged over its legitimate range of $-\infty \sim \infty$, yielding:

$$
\begin{aligned}
& f_{Z_{(k, p, l)(r)}}\left(z \mid H_{x}\right)=\int_{-\infty}^{\infty} f(\beta) \cdot f_{Z_{(k, p, l)(r)}}\left(z \mid H_{x}, \beta\right) d \beta \\
& =\int_{0}^{\infty}\left(\frac{e^{-\beta / \sigma^{2}}}{\sigma^{2}}\right) \cdot \frac{\exp \left[-\left(z+\beta \lambda_{x}\right) / 2\right]}{2} \\
& \cdot \mathcal{I}_{0}\left(\sqrt{\beta \lambda_{x} z}\right) d \beta \\
& =\frac{\exp \left[-z /\left(2+\lambda_{x} \sigma^{2}\right)\right]}{\left(2+\lambda_{x} \sigma^{2}\right)} \\
& \equiv \frac{\exp \left[-z /\left(2+\overline{\lambda_{x}}\right)\right]}{\left(2+\overline{\lambda_{x}}\right)} \text {, }
\end{aligned}
$$

where the effects of both timing errors and frequency mismatches are encapsulated by the definition of $\left(\overline{E_{c} / I_{0}}\right)^{\prime}$ and the corresponding non-centrality parameter, $\overline{\lambda_{x}} \equiv \lambda_{x} \sigma^{2}$ is either $2 N\left(\frac{\overline{E_{c}}}{I_{0}}\right)^{\prime}$ when the desired signal is deemed to be present $(x=$ $1)$ or $\frac{2}{N}\left(\frac{\overline{E_{c}}}{I_{0}}\right)^{\prime}$ when it is deemed to be absent $(x=0)$. We also define $\mu_{x}=\left(2+\overline{\lambda_{x}}\right)$, which physically represents a new biased non-centrality parameter. Further details on the related calculations and derivations can be found in [14], [15]. When using the procedures outlined in [14], finally, the decision variables $Z_{(k, p, l)}$ are constituted by the sum of $R$ number of independent variables according to $Z_{(k, p, l)}=\sum_{r=1}^{R} Z_{(k, p, l)(r)}$, whilst the desired signal's PDF may be formulated using the procedure outlined in [14], [15] as:

$$
f_{Z_{(k, p, l)}}\left(z \mid H_{x}\right)=\frac{z^{(R-1)} e^{-z / \mu_{x}}}{\Gamma(R) \cdot \mu_{x}^{R}},
$$

where $\Gamma(\cdot)$ is the Gamma function. Then, the $P_{D}$ or $P_{F}$ corresponding to $x=1$ or 0 , respectively, may be expressed as

$$
\begin{aligned}
\left.\left.P\right|_{\xi=D \text { or } F}\right|_{(\text {cooperative })} & =\int_{\theta}^{\infty} f_{Z_{(k, p, l)}}\left(z \mid H_{x}\right) d z \\
& =\exp \left(-\frac{\theta}{\mu_{x}}\right) \cdot \sum_{r=0}^{R-1} \frac{\left(\theta / \mu_{x}\right)^{r}}{r !}(10)
\end{aligned}
$$

where $\theta$ is a threshold value. On the other hand, in case of colocated MIMOs, the $P_{D}$ or $P_{F}$ corresponding to $x=1$ or 0 , respectively, is obtained as [14] - [16]

$$
\left.\left.P\right|_{\xi=D \text { or } F}\right|_{(\text {co-located })}=\exp \left(-\frac{\theta}{\mu_{x}}\right) \cdot \sum_{k=0}^{P R-1} \frac{\left(\theta / \mu_{x}\right)^{k}}{k !},
$$

By employing Eqs. 10 and 11, the transfer functions required for calculating the achievable MAT of both the fixed RS-aided scenarios and of the co-located one will be derived in the forthcoming section. 


\section{MAT ANALYSIS OF CODE ACQUISITION IN RS-AIDED SCENARIOS}

The length of the PN sequence in our system was also assumed to be $\left(2^{15}-1\right) \cdot T_{c}$, as in the DL of the intercell synchronous CDMA-2000 system [14], [15] for instance, where the chip-duration is $T_{c}=1 / 1.2288 \mu \mathrm{s}$. In the case of code acquisition contrived for the DS-CDMA DL, the main design goal is to acquire coarse timing of the first received signal path impinging at the receiver, since this timing information is used as that of the reference finger of the Rake receiver. In [10], [11], explicit MAT formulas were provided for a single-antenna aided serial search based code acquisition scheme. There is a difference between a singleand co-located MIMO-aided schemes as well as a RS-assisted one in terms of analysing the MAT, because a signal received from a RS is a time delayed version of that directly received from the BS. Hence the independently fading multiple signal replicas received from $\mathrm{RSs}$ cannot be directly combined, as opposed to the co-located MIMO-element scenarios [14] [16]. Groups of multi-path components received from a BS and RS(s) may or may not overlap, depending on the path delays experienced. However, for the sake of simplifying our analysis, we assume that every group of multi-path components is nonoverlapping and each arrives with a time delay that is an integer multiple of the chip-duration $\tau_{p}$, because the derivation of the exact MAT formula can be simplified without grave inaccuracies, when the uncertainty region may be assumed to be substantially wide, such as $\left(2^{15}-1\right)$ chips $^{3}$. In terms of deriving both the $P_{D}$ and the $P_{F}$ based upon invoking multiple receive antennas, we will commence our discourse by analysing the MAT performance of code acquisition schemes employing Double Dwell Serial Search (DDSS) [10] - [12], [14] - [16]. We assume that in each chip duration $T_{c}, \alpha$ number of correct timing hypotheses are tested instead of just a single one, which are spaced by $T_{c} / \alpha$. Hence the number of legitimate locking positions to be tested is increased by a factor of $\alpha$. Moreover, as mentioned in Section II, when the $L$ multi-path signals arrive at time delays, which are integer multiples of the chip-duration $\tau_{l}$, then the relative frequency of the signal being present is increased $L$-fold. Similarly, the relative frequency of the signal being present is increased by a factor of $P$, which is proportional to the total number of RSs and BS, $P$. The required transfer functions [10], [11], are defined as follows. The entire successful detection related transfer function $H_{D}(z)$ encompasses all the branches of a state diagram [10], [11], which lead to successful detection. Furthermore, $H_{0}(z)$ indicates the absence of the desired user's signal at the output of the acquisition scheme, whilst $H_{M}(z)$ represents the overall miss probability of a search run carried out across the entire uncertainty region. The related processes are detailed for DDSS in [11], [12], [14], [15]. Then, it may be shown that the generalised expression derived for computing

\footnotetext{
${ }^{3}$ To elaborate a little further, when the uncertainty region is $\left(2^{15}-1\right)$ chip durations, the ratio between the number of chips in the uncertainty region and that of the legitimate locking positions corresponding to the signal being absent and present, respectively, is at most on the order of $10^{-4}$, which allows us to employ our aforementioned assumptions in the derivation of the MAT formula.
}

the MAT of the DDSS scheme is given by [10], [17]:

$$
\begin{array}{r}
E\left[T_{A C Q}\right]=\frac{1}{H_{D}(1)}\left[H_{D}{ }^{\prime}(1)+H_{M}{ }^{\prime}(1)+\right. \\
\left.\left\{(\nu-2 \alpha L P)\left[1-\frac{H_{D}(1)}{2}\right]+\frac{1}{2} H_{D}(1)\right\} H_{0}{ }^{\prime}(1)\right] \cdot \tau_{D 1} \\
\approx \frac{\left(1+H_{M}(1)\right) \cdot H_{0}{ }^{\prime}(1)}{2 \cdot\left(1-H_{M}(1)\right)} \cdot\left(\nu \cdot \tau_{D 1}\right),
\end{array}
$$

where $\left.H_{x}^{\prime}(z)\right|_{x=D, M \text { or } 0}$ is a derivative of $\left.H_{x}(z)\right|_{x=D, M \text { or } 0, \quad \nu \text { represents the total number of }}$ uncertainty positions to be searched and $\tau_{D 1}$ denotes the $1^{\text {st }}$ dwell time. Again, the exact MAT formula can be simplified, if $\nu$ is significantly higher than the number of $H_{1}$ states, where $H_{1}$ represents that the signal may be deemed to be present [17]. Therefore, in order to simplify our numerical performance analysis, we adopted the specific approximation of the exact MAT expression proposed in [17]. Explicitly, since each resolvable path contributes two $H_{1}$ hypotheses and because the average $P_{D}$ associated with these two hypotheses is the same, the overall miss probability of the DDSS scheme in the fixed RS-aided scenario may be expressed as:

$$
\begin{aligned}
\left.H_{M}(1)\right|_{(\text {cooperative })}= & \prod_{\substack{p=1 \\
P_{l=1}}}^{L} \prod_{\zeta=1}^{\alpha}\left[\left(1-P_{D 1(p, l, \zeta)}\right)+\right. \\
& \left.P_{D 1(p, l, \zeta)} \cdot\left(1-P_{D 2(p, l, \zeta)}\right)\right]^{2}
\end{aligned}
$$

where $\left.P_{D x(p, l, \zeta)}\right|_{x=1, \text { or } 2}$ represent the $P_{D}$ of both the search and the verification modes of the DDSS arrangements, respectively. On the other hand, the overall miss probability of the DDSS scheme in the co-located MIMO scenario may be expressed as [14] - [16]:

$$
\begin{aligned}
\left.H_{M}(1)\right|_{(\text {co-located })}= & \prod_{l=1}^{L} \prod_{\zeta=1}^{\alpha}\left[\left(1-P_{D 1(l, \zeta)}\right)+\right. \\
& \left.P_{D 1(l, \zeta)} \cdot\left(1-P_{D 2(l, \zeta)}\right)\right]^{2}
\end{aligned}
$$

respectively, where $\left.P_{D x(l, \zeta)}\right|_{x=1, \text { or } 2}$ are the $P_{D}$ of both the search and the verification modes of the DDSS arrangements, respectively. The $H_{0}{ }^{\prime}(1)$ value of the DDSS scheme is expressed as:

$$
H_{0}{ }^{\prime}(1)=1+m \cdot P_{F 1}+K \cdot P_{F 1} \cdot P_{F 2},
$$

where $K$ denotes the false locking penalty factor expressed in terms of the number of chip intervals required by an auxiliary device for recognising that the code-tracking loop is still unlocked and $m$ represents the exponent of $z$ in the verification mode. Furthermore, $\left.P_{F x}\right|_{x=1 \text {, or } 2}$ represent the $P_{F}$ in both the search and in the verification mode of the DDSS scheme, respectively.

\section{NUMERICAL SYSTEM PERFORMANCE RESULTS}

In this section we will characterise the MAT performance of the cooperative MIMO aided DS-CDMA code acquisition scheme of Fig.2. In Table I we outlined the maximum SINR degradation imposed by both the Doppler shift and the frequency drift between the transmitter and receiver oscillators in 
TABLE I

MAXIMUM SINR DEGRADATION INFLICTED BY BOTH THE DOPPLER SHIFT AND A $1000 \mathrm{HZ}$ FREQUENCY DRIFT IN CONJUNCTION WITH THE COHERENT INTEGRATION INTERVAL OF $N$ CHIP DURATIONS AT A CARRIER FREQUENCY OF $1.9 \mathrm{GHZ}$

\begin{tabular}{|l|l|l|l|l|l|}
\hline N(Chips) & 64 & 128 & 256 & 384 & 512 \\
\hline Degradation $(\mathrm{dB})$ & 0.061 & 0.2449 & 0.9969 & 2.3144 & 4.3213 \\
\hline
\end{tabular}

conjunction with the coherent integration interval durations $\tau_{D}$ seen in the code acquisition schematic of Fig.2. The number of chips over which the integrator of Fig.2 sums the square of the envelope detector's output in both the search and the verification modes of DDSS is assumed to be 64 and 256 in the scenarios having one and two RSs as well as $R=1,2$ and 4 receive antennas. In the co-located MIMO scenario we assume having the same number of chips in both the search and the verification modes. The values of the link imbalance between the BS as well as the one RS or two RS aided scenario is assumed to be 0,3 or $6 \mathrm{~dB}$. Additionally, we assumed that there is no link imbalance between the two RSs. The spreading factor of the Walsh code to be acquired was selected to be 128 . The frequency drift was assumed to be $1000 \mathrm{~Hz}$ [10], whilst the carrier frequency was $1.9 \mathrm{GHz}$. As an example of a high mobile speed, it is reasonable to postulate $160 \mathrm{~km} / \mathrm{h}$. We also assumed that the sampling inaccuracy caused by having a finite, rather than infinitesimally low search step size of $\Delta=T_{c} / 2$ was $-0.91 \mathrm{~dB}$, which is a typical value for the search step size [10], [11]. Furthermore, in case of $\Delta=T_{c} / 2$, the effect of cell correlation becomes modest [24], hence it is reasonable to assume that two consecutive cells are uncorrelated. The total uncertainty region of code acquisition was assumed to entail $2 *\left(2^{15}-1\right)=65,534$ hypotheses. Finally, in the spirit of [11], the false locking penalty factor was assumed to be 1000 chip-durations. Both single-path and multi-path scenarios were considered. Each of the three paths of the multi-path scenario arrived with a relative time delay of one chip and they had a $3 \mathrm{~dB}$ lower magnitude for the first received path as well as $6 \mathrm{~dB}$ lower magnitude for the second and the third received paths than the LOS path of the single-path scenario, respectively. All paths were assumed to be present in a given search window. All the performance curves have been obtained at the optimum decision threshold of $E_{c} / I_{0}=-13 \mathrm{~dB}$ designed for the code acquisition scheme. The operational range of the twoRS scenario was assumed to be $3 \mathrm{~dB}$ lower than that of the single-RS one, because it is highly likely that the former may be situated near the cell-edge.

Fig. 3 characterises the MAT versus $E_{c} / I_{0}$ performance of the DDSS code acquisition arrangement parameterised with the number of paths for a single RS and for $P=2$ colocated transmit antennas. A BS having a single transmit antenna is also considered to be a benchmarker. In Figs. 3 to 7 , ' $L 1$ ' denotes a single-path scenario, whilst ' $L 3$ ' presents the scenario of encountering three paths. When considering Fig.3, ' $\left.P 2 R 1 L x\right|_{x=1 \text { or } 3}: 0 d B$ ' represents a cooperative transmission scenario (i.e., Scenario (1) of Fig.1), whereas 'P2R1Lx $\left.\right|_{x=1 \text { or } 3}$ ' indicates a co-located transmission scenario (i.e., Scenario (2) of Fig.1). Furthermore, in Figs.4 to

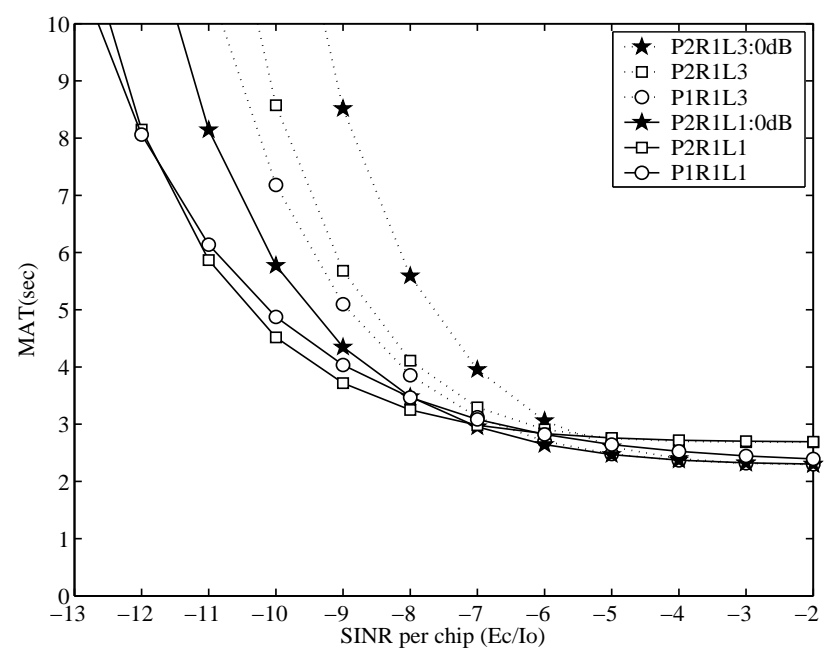

Fig. 3. MAT versus SINR per chip performance of the code acquisition system for DDSS parameterised with the number of paths for one RS as well as for co-located $P=1$ and 2 transmit antennas (normalised-power scenario).

7, the solid lines indicate the MAT curves recorded for RSaided transmissions, whereas the dotted lines represent the MAT curves of our benchmarker. The effects of the different link imbalance values of 0,3 , and $6 \mathrm{~dB}$ are clearly visible in all the figures. When considering the normalised-power scenario, the solid line denoted as ' $P 2 R 1 L 1$ ' in Fig.3 illustrates a similar MAT performance to the ' $P 1 R 1 L 1$ ' arrangement right across the entire $E_{c} / I_{0}$ range. The solid line denoted as 'P2R1L1 : $0 d B$ ' indicates the worst MAT performance among all the three cases considered, suggesting that the multipath diversity does not lead to any MAT performance gain. In case of the three-path scenario, the number of successful detection states was increased by a factor of $L=3$, but despite of this, the MAT performance of this three-path scenario became worse than that of the single-path one. The dotted line denoted as ' $P 1 R 1 L 3$ ' in Fig. 3 exhibits the best achievable MAT performance among all the three cases. This is because a low 'per-path-power' of the $P=2$ transmitter scenario leads to a low acquisition performance in the multi-path scenario. Even in case of 'P $P R 1 L 3$ ' we attain no diversity gain, because the performance degradation imposed by the low per-branch-power of both transmit and multi-path diversity becomes more drastic. Similarly to the single-path case, the ' $P 2 R 1 L 3: 0 d B$ ' scenario represents the worst performance. Accordingly, in the normalised-power scenario, sharing a given total transmit power at the BS by multiple transmit antennas becomes detrimental in terms of the MAT performance, in most practical scenarios. The related in-depth analysis can be found in [14] - [16]. The increased-power scenarios are analysed in Figs. 4 to 7.

Fig.4 illustrates the achievable MAT versus SINR per chip performance of the DDSS code acquisition scheme parameterised with both the grade of link imbalance and with the number of receive antennas for a single RS and a single propagation path. Observe in Fig.4 that when the link imbalance is decreased, we experience an improved MAT performance. In a case of having no link imbalance, the MAT 


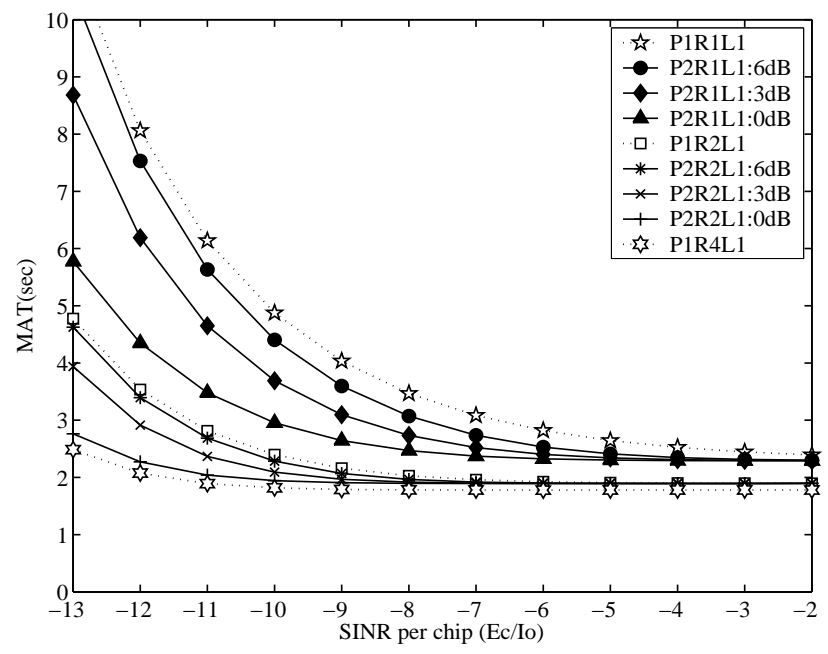

Fig. 4. MAT versus SINR per chip performance of the code acquisition system for DDSS parameterised with link imbalance and the number of receive antennas for one RS and a single path (increased-power scenario).

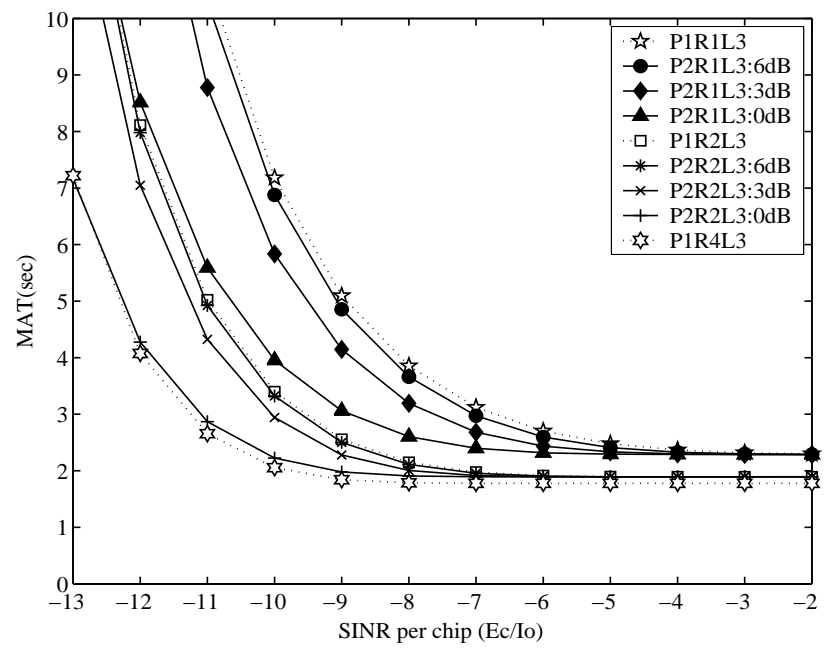

Fig. 5. MAT versus SINR per chip performance of the code acquisition system for DDSS parameterised with link imbalance and the number of receive antennas for one RS and three paths (increased-power scenario).

performance approaches that of having two receive antennas. On the other hand, in case of having a $6 \mathrm{~dB}$ imbalance, only a marginal diversity gain is achieved, hence the attainable MAT performance improvement also becomes negligible. Fig. 5 characterises the MAT versus SINR per chip performance of the code acquisition scheme having exactly the same parameters as those considered in Fig.4, except for the scenario having three paths. Similarly to the conclusions of Fig.4, as the link imbalance is decreased, all the curves seen in Fig.5 indicate an improved MAT performance and vice versa. The abovementioned conclusions explicitly demonstrate that employing a single RS is beneficial in terms of the achievable MAT performance, and as expected, the achievable improvements depend on the value of the link imbalance, regardless whether singlepath or multi-path scenarios are considered. However, using a single RS cannot guarantee maintaining a high diversity gain due to the fluctuation of the RS's link quality.

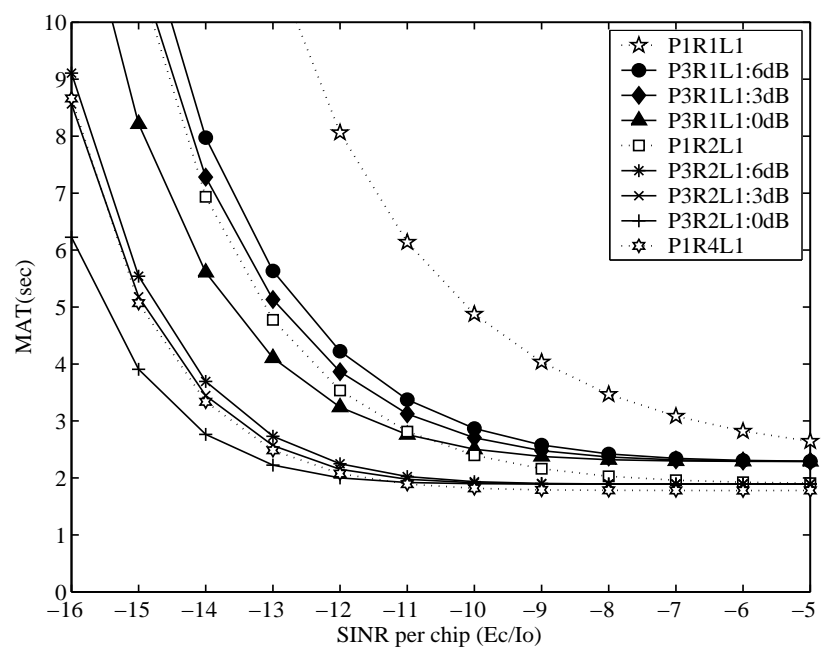

Fig. 6. MAT versus SINR per chip performance of the code acquisition system for DDSS parameterised with link imbalance and the number of receive antennas for two RSs and a single path (increased-power scenario).

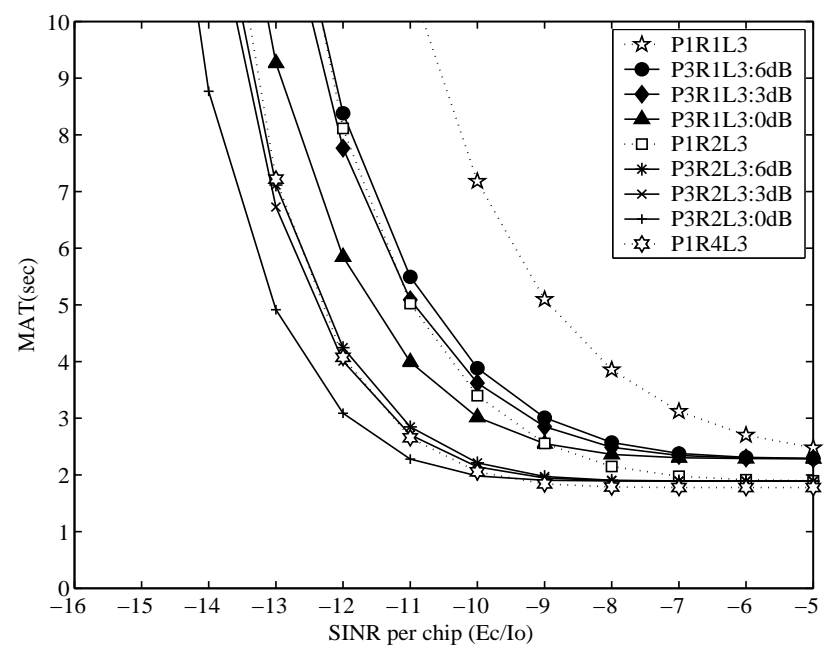

Fig. 7. MAT versus SINR per chip performance of the code acquisition system for DDSS parameterised with link imbalance and the number of receive antennas for two RSs and three paths (increased-power scenario).

For comparison, Figs.6 and 7 characterise the achievable MAT versus SINR per chip performance of the DDSS code acquisition scheme parameterised with both the value of link imbalance and the number of receive antennas, when considering two RSs in both a single-path (Fig.6) and in a three-path scenario (Fig.7), respectively. The results of Figs.6 and 7 also exhibit similar MAT performance trends, as shown in Figs.4 and 5, although the degree of achievable performance improvements is higher than that of the single-RS scenario. To elaborate on the above results a little further, in case of having a $0 \mathrm{~dB}$ imbalance the MAT performance attained becomes better than that of the benchmarker supported by $R=2$ or 4 receive antennas but no RS. Even a link imbalance of 6 $\mathrm{dB}$ is capable of ensuring a similar performance to that of the benchmarker. Therefore, owing to the random fluctuation of the link quality, the employment of two RSs might be beneficial in order to achieve a substantial performance 
improvement in RS-aided scenarios. In comparison to the detrimental effects of distributing the total transmit power over both several co-located and cooperative transmit antennas, as shown in Fig.3 and [14] - [16], based upon Figs.4 to 7 characterising the increased-power scenario, we infer that RSaided scenarios benefit from higher multi-path diversity gains. In case of employing multiple co-located receive antennas at the MS, an additional receiver diversity gain is also achieved. Accordingly, by exploiting an appropriate combination of RS-aided transmissions and multiple co-located MS receive antennas, in the scenarios considered our scheme is capable of attaining a better performance than the benchmarker scheme benefitting from having four co-located MS receive antennas. It is worth mentioning furthermore that increasing the number of RSs used explicitly increases the number of independently fading multi-path components by a factor of $P$. However, this assumption implicitly expects that the extra transmit power required can indeed be provided by the RS. It is also noted that in practice the size-limitation of the MS restricts the number of DL receive antennas. In order to exploit the beneficial characteristics of cooperative transmissions, assigning additional power to each additional transmit antenna becomes inevitable during the initial code acquisition stage. The importance of post-initial acquisition [14] - which is capable of identifying the timing instants of the affordable-complexity-dependent number of delayed and independently fading received signal paths to be combined by a Rake receiver - is also worth exploring further. This is particularly so in the code acquisition schemes designed for ultra-wide band systems, owing to the inherent presence of an extremely high number of multi-path components [25].

\section{CONCLUSION}

In this paper we considered RS-aided distributed DL MIMOs and the benefits of receive diversity in code acquisition schemes operating in the inter-cell synchronous CDMA DL. In contrast to the detrimental effects of sharing the total transmit power across multiple transmit antennas in both co-located and cooperative MIMO element based scenarios, as described in Fig.3 and in [14] - [16], our analysis based on Figs.4 to 7 explicitly shows that in the absence of link imbalance, the achievable MAT performance of RS-aided distributed DL MIMOs approaches that of classic receive diversity schemes benefitting for multiple independently fading MS receive antenna elements, when considering the same total diversity order. Naturally, the performance of the acquisition schemes employing multiple co-located transmit antenna elements erodes in the presence of correlated shadow fading, whilst that of the RSaided scheme is expected to remain unaffected, provided that the RS is sufficiently far from the MS. On the other hand, in a scenario of having a high link imbalance, only marginal MAT performance gains may be achieved, regardless whether single-path or multi-path propagation scenarios are considered. Hence for the sake of exploiting the diversity benefits of RSaided transmissions, the employment of at least two RSs might be recommended. When additionally invoking multiple colocated and yet independently fading receive antennas at the MS, further diversity gains may be achieved.

\section{ACKNOWLEDGEMENT}

The financial support of the Ministry of Information and Communication (MIC), Republic of Korea, of the EPSRC, UK and of the EU is gratefully acknowledged.

\section{REFERENCES}

[1] V. Tarokh, A. Naguib, N. Seshadri, and A. R. Calderbank, SpaceTime Codes for High Data Rate Wireless Communication: Performance Criteria in the Presence of Channel Estimation Errors, Mobility, and Multiple Paths, IEEE Transactions on Communications, vol. 47, No. 2, 1999, pp199-207.

[2] D. Gesbert, M. Shafi, D.S. Shiu, P.J. Smith, and A. Naguib, From Theory to Practice: An Overview of MIMO Space-Time Coded Wireless Systems, IEEE Journal on Selected Areas in Communications, vol. 21, No.3, 2003, pp281-302.

[3] S.X. Ng, L. Hanzo, On the MIMO Channel Capacity of Multidimensional Signal Sets, pp528-536, IEEE Transactions on Vehicular Technology, vol. 55, No. 2, 2006.

[4] A. Sendonaris, E. Erkip and B. Aazhang, User Cooperation Diversity-Part I: System Description, IEEE Transactions on Communications, vol. 51, No. 11, 2003, pp1927-1938.

[5] J. N. Laneman, D. N. C. Tse and G. W. Wornell, Cooperative Diversity in Wireless Networks: Efficient Protocols and Outage Behavior, IEEE Transactions on Information Theory, vol. 50, No. 12, 2004, pp3062-3080.

[6] R. Pabst, B. H. Walke, D. C. Schultz, P. Herhold, H. Yanikomeroglu, S. Mukherjee, H. Viswanathan, M. Lott, W. Zirwas, M. Dohler, H. Aghvami, D. D. Falconer and G. P. Fettweis, Relay-based Deployment Concepts for Wireless and Mobile Broadband Radio, IEEE Communications Magazine, vol. 42, No. 9, 2004, pp80-89.

[7] L. Long and E. Hossain, Multihop Cellular Networks: Potential Gains, Research Challenges, and a Resource Allocation Framework, IEEE Communications Magazine, vol. 45, No. 9, 2007, pp66-73.

[8] D. Soldani and S. Dixit, Wireless Relays for Broadband Access, IEEE Communications Magazine, vol. 46, No. 3, 2008, pp5866.

[9] L-L Yang, L. Hanzo, Serial Acquisition Performance of SingleCarrier and Multicarrier DS-CDMA over Nakagami-m Fading Channels, IEEE Transactions on Wireless Communications, vol. 1, No. 4, 2002, pp692-702.

[10] A.J. Viterbi, CDMA: Principles of Spread Spectrum Communication, Chapter 3, Addison-Wesley, 1995.

[11] H.R. Park, Performance Analysis of a Double-Dwell Serial Search Technique for Cellular CDMA Networks in the Case of Multiple Pilot Signals, IEEE Transactions on Vehicular Technology, vol. 48, No. 6, 1999, pp1819-1830.

[12] M.K. Simon, J.K. Omura, R.A. Scholtz and B.K. Levitt, Spread Spectrum Communications Handbook, McGraw-Hill Professional, 2001

[13] L. Hanzo, L-L Yang, E-L Kuan, K. Yen, Single- and MultiCarrier DS-CDMA, IV Multi-Carrier CDMA, Chapter 21, Initial Synchronization of DS-CDMA and MC-CDMA Systems, Wiley, 2003.

[14] S.H. Won and L. Hanzo, Initial and Post-Initial Acquisition in the Serial Search Based Noncoherent Multiple Transmit/Receive Antenna Aided DS-CDMA Downlink, Vehicular Technology Conference, vol. 5, 2006, pp2246-2250.

[15] S.H. Won and L. Hanzo, Differentially Coherent Code Acquisition in the MIMO-aided Multi-carrier DS-CDMA Downlink, IET Communications, vol. 1, Issue 4, 2007, pp662-670.

[16] S.H. Won and L. Hanzo, Analysis of Serial-Search-Based Code Acquisition in the Multiple-Transmit/Multiple-ReceiveAntenna-Aided DS-CDMA Downlink, IEEE Transactions on Vehicular Technology, vol. 57, No. 2, 2008, pp1032-1039. 
[17] L-L Yang, L. Hanzo, Serial Acquisition of DS-CDMA Signals in Multipath Fading Mobile Channels, IEEE Transactions on Vehicular Technology, vol. 50, No. 2, 2001, pp617-628.

[18] J.G. Proakis, Digital Communications, 4th ed. Chapter 2, McGraw-Hill, 2001, pp17-79.

[19] G.E. Corazza, C. Caini, A. Vanelli-Coralli, A. Polydoros, DSCDMA Code Acquisition in the Presence of Correlated FadingPart I: Theoretical Aspects, IEEE Transactions on Communications, vol. 52, No. 7, 2004, pp1160-1168.

[20] R.N. McDonough, A.D. Whalen, Detection of Signals in Noise, 2nd ed. Chapter 5, Academic Press, Inc., 1995.

[21] S. Kim, Effect of Spatial Fading Correlation on CDMA CodeAcquisition Performance, IEE Proceedings - Communication, Vol.152, No. 1, 2005, pp103-112.

[22] L. Hanzo and M.Münster and B.J. Choi and T.Keller, OFDM and MC-CDMA for Broadcasting Multi-User Communications, WLANs and Broadcasting, John Wiley \& Sons, 2003.

[23] J-C Lin, Differentially Coherent PN Code Acquisition with FullPeriod Correlation in Chip-Synchronous DS/SS Receivers, IEEE Transactions on Communications, vol. 50, No. 5, 2002, pp698702.

[24] W.-H. Sheen, J.-K. Tzeng and C.-K. Tzou, Effects of Cell Correlations in A Matched-Filter PN Code Acquisition for Direct-Sequence Spread-Spectrum Systems, IEEE Transactions on Vehicular Technology, vol. 48, No. 3, 1999, pp724-732.

[25] I. Ramachandran and S. Roy, On Acquisition of Wideband Direct-sequence Spread Spectrum Signals, IEEE Transactions on Wireless Communications, vol. 5, No. 6, 2006, pp1537-1546.

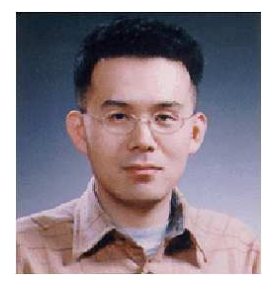

SeungHwan Won (M'04) received the B.S. and M.S. degrees in Radio Science and Engineering from Korea University, Seoul, Republic of Korea, in 1999 and 2001, respectively. He was a research engineer in Mobile Communication Technology Research Lab, LG Electronics R\&D, from January 2001 to September 2004. He was the recipient of the 2004 state scholarship of the Information and Telecommunication National Scholarship Program, Ministry of Information and Communication (MIC), Republic of Korea. Since October 2004, he has been working towards the Ph.D. degree in the Communications Research Group, School of Electronics and Computer Science at the University of Southampton, UK. His major research interests include initial synchronization in non-coherent MIMO aided single- and multi-carrier CDMA, DS-UWB and OFDMA as well as in iterative synchronization schemes designed for MIMO aided single- and multi-carrier transmission systems.

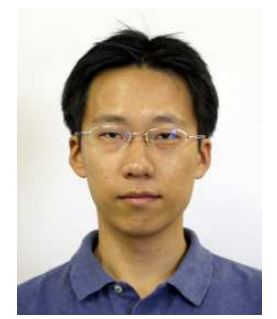

Kyungchun Lee received the B.S., M.S. and $\mathrm{Ph} . \mathrm{D}$. degrees in electrical engineering from Korea Advanced Institute of Science and Technology (KAIST), Daejeon, in 2000, 2002, and 2007, respectively. From September 2005 until February 2006, he was a visiting research student with the Communication Research Group, University of Southampton, U.K, where he held a post-doctorial visiting research fellowship from April 2007 until June 2008. Currently, he is with Samsung Electronics, P.O. Box 105, Suwon, Korea. His research interests include signal processing for wireless communications with focus on MIMO and relay systems.

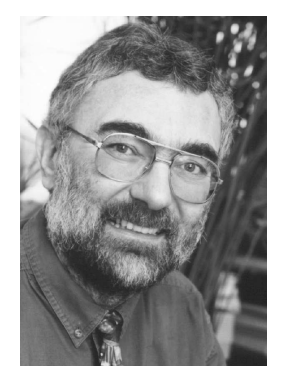

Lajos Hanzo, Fellow of the Royal Academy of Engineering, received his first-class degree in electronics in 1976 and his doctorate in 1983. In 2004 he was awarded the Doctor of Sciences (DSc) degree by the Uniersity of Southampton, UK. During his career in telecommunications he has held various research and academic posts in Hungary, Germany and the UK. Since 1986 he has been with the Department of Electronics and Computer Science, University of Southampton, UK, where he holds the chair in telecommunications. He co-authored 15 John Wiley and IEEE Press books totalling 10000 pages on mobile radio communications, published about 700 research papers, organised and chaired conference conferences, presented various keynote and overview lectures and has been awarded a number of distinctions. Currently he heads an academic research team, working on a range of research projects in the field of wireless multimedia communications sponsored by industry, the Engineering and Physical Sciences Research Council (EPSRC) UK, the European IST Programme and the Mobile Virtual Centre of Excellence (VCE), UK. He is an enthusiastic supporter of industrial and academic liaison and he offers a range of industrial courses. Lajos is also an IEEE Distinguished Lecturer of both the Communications as well as the Vehicular Technology Society, a Fellow of both the IEEE and the IEE. He is an editorial board member of the Proceedings of the IEEE and a Governer of the IEEE VT Society. For further information on research in progress and associated publications please refer to http://www-mobile.ecs.soton.ac.uk 